\title{
Avaliação radiográfica da descompensação do tronco após artrodese seletiva torácica em portadores de escoliose idiopática do adolescente King II (Lenke B e C)
}

Radiographic evaluation of trunk decompensation with selective thoracic arthrodesis in patients with adolescent idiopathic scoliosis type King II (Lenke B e C)

Evaluación radiográfica de la descompensación del tronco después de una artrodesis selectiva torácica en portadores de escoliosis idiopática del adolescente King II (Lenke B y C)

\author{
Osmar Avanzi ${ }^{1}$ \\ Elcio Landim ${ }^{2}$ \\ Robert Meves ${ }^{3}$ \\ Maria Fernanda Silber Cafaro ${ }^{4}$ \\ Ricardo Shigueaki Galhego Umeta ${ }^{5}$ \\ Jose Thiago Portella Kruppa ${ }^{6}$
}

\section{RESUMO}

Objetivo: avaliar a incidência de descompensação radiográfica do tronco após a artrodese seletiva torácica com instrumental de $3^{\mathrm{a}}$ geração na escoliose idiopática do adolescente (EIA) King II (Lenke B e C) após acompanhamento mínimo de um ano. Métodos: foram avaliadas retrospectivamente as radiografias pré-operatórias, pós-operatórias imediatas e do último acompanhamento de 22 pacientes tratadas no período de 1993 a 2007. Observou-se a porcentagem de correção da curva torácica e lombar pelo método de Cobb, e o balanço coro-

\section{ABSTRACT}

Objective: to evaluate radiographic results regarding trunk decompensation of AIS treated with selective thoracic arthrodesis and $3^{a}$ generation instrumentation. Methods: preoperatory, immediately post-operatory and last radiography evaluations were carried out in 22 patients with adolescent idiopathic scoliosis AIS type King II treated with selective thoracic arthrodesis from 1993 to 2007. The percentage of correction of the thoracic and lumbar curve was observed by means of Cobb method, as well as the trunk coronal balance by using SRS
\end{abstract}

\section{RESUMEN}

Objetivo: evaluar la incidencia de descompensación radiográfica del tronco después de una artrodesis selectiva torácica con instrumental de tercera generación en la escoliosis idiopática del adolescente (EIA) King II (Lenke $B$ y $C$ ), después de un acompañamiento mínimo de un año. Métodos: fueron evaluados retrospectivamente las radiografias preoperatorias, postoperatorias inmediatas y del último acompañamiento de 22 pacientes tratados en el periodo de 1993 a 2007. Se observó un porcentaje de correlación de la curva torácica y lumbar, por el método de

\footnotetext{
Faculdade de Ciências Médicas da Santa Casa de São Paulo - FCMSCSP - São Paulo (SP), Brasil.

'Professor Adjunto do Departamento de Ortopedia e Traumatologia da Faculdade de Ciências Médicas da Santa Casa de São Paulo - FCMSCSP - São Paulo (SP), Brasil.

${ }^{2}$ Consultor Sênior do Grupo de Coluna do Departamento de Ortopedia e Traumatologia da Faculdade de Ciências Médicas da Santa Casa de São Paulo FCMSCSP - São Paulo (SP), Brasil.

${ }^{3}$ Professor-assistente do Departamento de Ortopedia e Traumatologia da Irmandade Santa Casa de Misericórdia de São Paulo - ISCMSP - São Paulo (SP), Brasil. ${ }^{4}$ Assistente e Professora Instrutora do Grupo de Coluna do Departamento de Ortopedia e Traumatologia da Irmandade Santa Casa de Misericórdia de São Paulo ISCMSP - São Paulo (SP), Brasil.

${ }^{5}$ Pós-Graduando (Mestrado) do Grupo de Coluna do Departamento de Ortopedia e Traumatologia da Faculdade de Ciências Médicas da Santa Casa de São Paulo - FCMSCSP - São Paulo (SP), Brasil.

${ }^{6}$ Médico Estagiário do Grupo de Coluna do Departamento de Ortopedia e Traumatologia da Faculdade de Ciências Médicas da Santa Casa de São Paulo FCMSCSP - São Paulo (SP), Brasil. 
nal do tronco do início e do final do acompanhamento foi avaliado pelos critérios da Scoliosis Research Society. Resultados: após correção média da curva torácica de $56 \pm 11 \%$ e da lombar de $49 \pm 13 \%$, verificamos descompensação coronal imediata em seis pacientes $(27,20 \%)$. Após acompanhamento médio de 65 meses, quatro pacientes $(18,18 \%)$ estavam com o tronco descompensado. Em apenas um paciente foi necessária extensão da artrodese, incluindo a curva lombar. Conclusões: a descompensação grave do tronco com necessidade de segundo procedimento para artrodese lombar foi complicação não frequente nesta série de casos. criteria. Results: after mean percentage of correction in thoracic curve of $56 \pm 11 \%$ and $49 \pm 13 \%$ in lumbar curve, we observed an immediate coronal decompensation in six patients (27.20\%). After a mean 65-month follow-up, four patients (18.18\%) showed trunk coronal decompensation. Only one patient needed a new surgery with arthrodesis extension, including the lumbar curve, due to lumbar curve decompensation. Conclusions: severe trunk decompensation in need of a second procedure for lumbar fusion was not a frequent complication in this series.

KEYWORDS: Scoliosis/surgery; Spinal fusion; Adolescent
Cobb, y el balance coronal del tronco al inicio y al final del acompañamiento por los criterios de la Scoliosis Research Society. Resultados: después de la corrección promedio de la curva torácica de $56 \pm 11 \%$ y de la lumbar de $49 \pm 13 \%$, se verificó descompensación coronal inmediata en seis pacientes $(27,20 \%)$. Después de acompañamiento promedio de 65 meses, cuatro pacientes $(18,18 \%)$ estaban con el tronco descompensado. En sólo un paciente fue necesaria una extensión de la artrodesis, incluyendo la curva lumbar. Conclusiones: la descompensación grave del tronco con necesidad de un segundo procedimiento para artrodesis lumbar fue una complicación no frecuente en esta serie de casos.

DESCRIPTORES: Escoliosis/ cirugía; Fusión vertebral; Adolescente Adolescente

\section{INTRODUÇÃO}

Dos diferentes tipos de escoliose idiopática do adolescente (EIA), o tratamento da curva torácica principal com lombar flexível (King II) é a mais controversa em função do risco de descompensação do tronco. Em teoria, a curva lombar compensatória se acomodará espontaneamente em relação à posição da curva torácica corrigida. Portanto, atinge-se o objetivo principal do tratamento operatório: correção parcial da curva para obter um tronco compensado com poucos níveis de artrodese. Apesar disso, observou-se descompensação coronal do tronco após a cirurgia, particularmente após o advento dos implantes de terceira geração ${ }^{1-4}$. Alguns autores atribuem esta complicação à falha na identificação da dupla curva verdadeira e à hipercorreção da curva torácica principal, prejudicando a capacidade compensatória da curva lombarr-5.

Em 1983, King et al. ${ }^{6}$ descreveram uma classificação para ajudar na identificação dos tipos de curvas que poderiam ser tratadas por intermédio da artrodese seletiva. Recomendaram, com base nas radiografias dinâmicas em inclinação de pacientes operadas com implante de segunda geração, que as curvas King II (curva torácica principal com lombar compensatória) fossem tratadas com artrodese somente da curva torácica.

Em razão do aumento da incidência de descompensação coronal do tronco após a utilização da instrumentação de $3^{\text {a }}$ geração, Lenke et al. ${ }^{7}$ publicaram outros critérios, como os modificadores lombares e o valor limite de flexibilidade de 25 graus, para auxiliar a definição do nível de artrodese e diminuir o risco de descompensação pósoperatória do tronco. Mesmo assim, a controvérsia a respeito da realização ou não da artrodese seletiva torácica nas curvas King II permanece ${ }^{8-14}$.

Dessa forma, o objetivo deste estudo foi avaliar os resultados radiográficos no que concerne à descompensação do tronco em portadores de EIA King II (Lenke B e C) tratados por artrodese seletiva torácica com instrumental de $3^{\mathrm{a}}$ geração em curvas.

\section{MÉTODOS}

Foram avaliadas retrospectivamente, após período um mínimo de acompanhamento de 12 meses, as radiografias dos pacientes portadores de EIA tipo King $\mathrm{II}^{6}\left(\right.$ Lenke $^{14} \mathrm{~B}$ e C) submetidos à artrodese seletiva torácica (limite inferior da artrodese até a primeira vértebra lombar), com implante metálico de $3^{\text {a }}$ geração realizado no período de 1993 a 2007, após aprovação do Comitê de Ética em Pesquisa da SCMSP.

As radiografias ortostáticas panorâmicas pré-operatórias nas incidências de anteroposterior (AP), de perfil (P) e AP com inclinações laterais em posição supina foram avaliadas. Os pacientes selecionados foram também classificados de acordo com os critérios de Lenke et al. ${ }^{14}$, sendo: oito $1 \mathrm{BN}$, um $1 \mathrm{~B}+, 111 \mathrm{CN}$, um $1 \mathrm{C}+\mathrm{e}$ um $2 \mathrm{CN}$. Onze pacientes foram submetidas ao instrumental de Cotrel-Dubousset com ganchos e em 11 a USS com montagem híbrida (parafusos pediculares no segmento distal da instrumentação e ganchos na parte proximal). Os dois momentos de observação foram 
no momento pós-operatório inicial (até uma semana após a cirurgia) e no último acompanhamento.

Vinte e dois pacientes preencheram os critérios de inclusão para o estudo, sendo 2 do sexo masculino e 20 do sexo feminino. A média de idade foi $14,63 \pm 2,47$ anos.

Realizamos as medidas das curvas conforme o método de $\mathrm{Cobb}^{15}$. A curva torácica principal média foi de $56,61 \pm 7,59$, e a curva lombar foi de $40,4 \pm 8,51^{\circ}$. No plano sagital, a cifose de T5-T12 foi em média de 25,04 $\pm 11,64^{\circ}$. No pós-operatório imediato, obteve-se correção em média da curva torácica para $25,04 \pm 7,37^{\circ}$, com uma porcentagem de correção de $56 \pm 11 \%$. Quanto à curva lombar, obteve-se uma melhora em média para $20,77 \pm 7,17^{\circ}$, com porcentagem de correção de $49 \pm 13 \%$. As radiografias do último acompanhamento mostraram perda média de correção de $15 \pm 13 \%$ da curva torácica e melhora de $1 \pm 23 \%$ da curva lombar.

A descompensação coronal do tronco foi determinada de acordo com os critérios da Scoliosis Research Society (SRS). Conforme a SRS, a descompensação coronal do tronco foi definida quando a distância da linha média de C7 com a linha sacral média era maior do que $20 \mathrm{~mm}$. Ambas as medidas foram utilizadas para definir resultado radiográfico satisfatório no que concerne à descompensação coronal do tronco ${ }^{16}$.

As variáveis categóricas foram descritas como frequências, e as numéricas, pelas medidas de resumo.

\section{RESULTADO}

No pré-operatório, o balanço coronal foi em média de 5,86 $\mathrm{mm}( \pm 11,80)$ para a esquerda. No pós-operatório imediato, o balanço coronal obtido foi em média de $12,95 \mathrm{~mm}$ $( \pm 14,65)$ para a esquerda, sendo seis descompensados para a esquerda, conforme os critérios da SRS. O balanço coronal final foi em média de $10,04 \mathrm{~mm}( \pm 13,34)$ para a esquerda. No último acompanhamento ambulatorial, foi constatado que o balanço coronal final foi em média de $10,04 \mathrm{~mm}( \pm 13,34)$ para a esquerda (Figura 1$)$, sendo que quatro permaneceram descompensados para a esquerda (Tabela 1). Do total de descompensados, um deles necessitou, por motivos estéticos, de novo procedimento cirúrgico para extensão da artrodese da curva lombar.
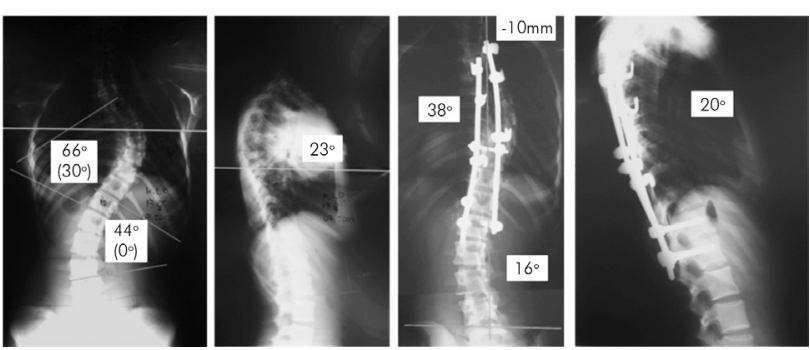

Figura 1

Paciente do sexo feminino, 13 anos de idade. À esquerda, radiografias AP e $P$ pré-operatórias. À direita, 6 anos após a cirurgia, exemplificando bom resultado da artrodese seletiva torácica, obtendo um bom balanço coronal (-10 mm).
TABELA 1 - Medidas de resumo dos portadores de EIA segundo a compensação do tronco durante o pós-operatório precoce e ao final do acompanhamento

\begin{tabular}{lcccc}
\hline Pós-operatório & Mínimo & Máximo & Médio & $\begin{array}{c}\text { Desvio } \\
\text { padrão }\end{array}$ \\
\hline Imediato & $-45 \mathrm{~mm}$ & $14 \mathrm{~mm}$ & $-12,95 \mathrm{~mm}$ & 14,65 \\
Final & $-35 \mathrm{~mm}$ & $14 \mathrm{~mm}$ & $-10,04 \mathrm{~mm}$ & 13,34 \\
\hline
\end{tabular}

\section{DISCUSSÃO}

Um dos objetivos do tratamento cirúrgico da EIA é corrigir a deformidade com balanço coronal e sagital, ao mesmo tempo deixando livre da artrodese o maior número de segmentos possíveis para evitar complicações como a doença articular degenerativa dolorosa precoce dos segmentos não-artrodesados, em particular na região lombar ${ }^{1-3,7,9}$. A aplicação do conceito da artrodese seletiva torácica permite controlar e corrigir parcialmente a curva torácica principal, mantendo a mobilidade dos segmentos lombares. Porém, em alguns casos, a curva lombar não é capaz de se acomodar à correção da curva torácica, levando à descompensação do tronco no plano coronal. Essa descompensação é mais frequentemente descrita nos pacientes portadores de EIA tipo King II $^{2,7,10,11}$. As causas mais comuns dessa complicação são atribuídas à identificação errada do tipo de curva (dupla curva verdadeira) ou à hipercorreção da curva torácica principal, prejudicando a capacidade compensatória da curva lombar ${ }^{1-5}$.

Em 1983, King et al. ${ }^{6}$ descreveram uma classificação para a identificação dos tipos de curvas que poderiam ser tratadas com artrodese seletiva . Eles recomendaram, com base no tratamento cirúrgico da escoliose com instrumental de $2^{\mathrm{a}}$ geração, que as curvas King II (curva torácica principal-lombar compensatória) fossem tratadas com artrodese somente da curva torácica.

Com o surgimento do instrumental de $3^{a}$ geração, com maior potencial de correção, reportam-se descrições de descompensação coronal para a esquerda ${ }^{1,3,7,9,11,16-19}$. Lenke et al. ${ }^{14}$ constatam, em uma série de casos de EIA tratados com instrumental de $3^{\mathrm{a}}$ geração, que a relação entre a curva torácica e a lombar sugerem fatores indicativos desta complicação e preconizam modificadores lombares para qualificar melhor a curva lombar. Os autores descrevem uma nova classificação para EIA que quantifica especificamente os aspectos estruturais de cada curva (torácica proximal, torácica principal e toracolombar/lombar) da escoliose. Eles estabeleceram, ainda, critérios estruturais para considerar uma curva principal estruturada ou compensatória não-estruturada. De acordo com esse sistema, a escoliose é classificada com base em: 1) tipo de curva; 2) translação da vértebra apical lombar; e 3) alinhamento sagital. Vários autores utilizam estes critérios para categorizar mais precisamente a amostra para análise dos resultados, argumentando que somente curvas consideradas estruturadas devem ser incluídas na artrodese ${ }^{1-9,14}$. 
Richards et al. ${ }^{10}$, em 1992, com 24 pacientes com EIA King II, observam que a capacidade de correção espontânea da curva lombar compensatória após esse procedimento ocorre na região proximal da curva lombar, porque a inclinação de L4 em relação à pelve manteve-se praticamente inalterada após a artrodese seletiva. Durante o acompanhamento, a correção espontânea adaptativa da curva lombar após a artrodese seletiva torácica pode justificar a melhora da compensação do tronco em algumas pacientes. Schwender et al. ${ }^{11}$ e Jansen et al..$^{13}$ descrevem a mesma constatação em suas respectivas séries de casos. Vale lembrar que duas pacientes da série aqui descrita apresentaram essa melhora gradual nos primeiros 12 meses após a cirurgia.

Não foi possível determinar valores ou sinais radiográficos preditivos da descompensação coronal com base nos achados da literatura, por isso muitos autores ainda utilizam a experiência clínica adquirida no trato desta afecção, em especial a observação da giba, para determinar a necessidade de inclusão da curva lombar na $\operatorname{artrodese}^{20}$.

\section{CONCLUSÃO}

A presente série sugere que o tratamento da escoliose idiopática do adolescente tipo King II com a artrodese torácica seletiva propicia uma boa correção e manutenção da correção das curvas torácica e lombar com balanço coronal final satisfatório. Algumas pacientes podem apresentar melhora gradual dessa descompensação, o que justifica conduta expectante em alguns casos descompensados. Porém, a descompensação coronal do tronco é uma complicação possível. Critérios radiográficos preditivos ainda precisam ser definidos para que esta complicação seja evitada.

\section{REFERÊNCIAS}

1. Chang KW, Chang KI, Wu CM. Enhanced capacity for spontaneous correction of lumbar curve in the treatment of major thoraciccompensatory $\mathrm{C}$ modifier lumbar curve pattern in idiopathic scoliosis. Spine (Phila Pa 1976). 2007;32(26):3020-9.

2. Edwards CC 2nd, Lenke LG, Peelle M, Sides B, Rinella A, Bridwell $\mathrm{KH}$. Selective thoracic fusion for adolescent idiopathic scoliosis with $\mathrm{C}$ modifier lumbar curves: 2- to 16-year radiographic and clinical results. Spine (Phila Pa 1976). 2004;29(5):536-46.

3. Dobbs MB, Lenke LG, Walton T, Peelle M, Della Rocca G, Steger-May $\mathrm{K}$, et al. Can we predict the ultimate lumbar curve in adolescent idiopathic scoliosis patients undergoing a selective fusion with undercorrection of the thoracic curve? Spine (Phila Pa 1976). 2004;29(3):277-85.

4. Frez R, Cheng JC, Wong EM. Longitudinal changes in trunkal balance after selective fusion of King II curves in adolescent idiopathic scoliosis. Spine (Phila Pa 1976). 2000;25(11):1352-9.

5. McCance S, Denis F, Lonstein JE, Winter RB. Coronal and sagittal balance in surgically treated adolescent idiopathic scoliosis with the King II curve pattern. A review of 67 consecutive cases having selective thoracic arthrodesis. Spine (Phila Pa 1976). 1998;23(19):2063-73.
6. King HA, Moe JH, Bradford DS, Winter RB. The selection of fusion levels in thoracic idiopathic scoliosis. J Bone Joint Surg Am. 1983;65(9):130213.

7. Lenke LG, Bridwell KH, Baldus C, Blanke K. Preventing decompensation in King type II curves treated with Cotrel-Dubousset instrumentation. Strict guidelines for selective thoracic fusion. Spine (Phila Pa 1976). 1992;17(8 Suppl):S274-81.

8. Newton PO, Faro FD, Lenke LG, Betz RR, Clements DH, Lowe TG, et al. Factors involved in the decision to perform a selective versus nonselective fusion of Lenke $1 \mathrm{~B}$ and 1C (King-Moe II) curves in adolescent idiopathic scoliosis. Spine (Phila Pa 1976). 2003;28(20):S217-23.

9. Lenke LG, Edwards CC 2nd, Bridwell $\mathrm{KH}$. The Lenke classification of adolescent idiopathic scoliosis: how it organizes curve patterns as a template to perform selective fusions of the spine. Spine (Phila $\mathrm{Pa}$ 1976). 2003;28(20):S199-207.

10.Richards BS. Lumbar curve response in type II idiopathic scoliosis after posterior instrumentation of the thoracic curve. Spine (Phila Pa 1976). 1992;17(8 Suppl):S282-6.

11. Schwender JD, Denis F. Coronal plane imbalance in adolescent idiopathic scoliosis with left lumbar curves exceeding 40 degrees: the role of the lumbosacral hemicurve. Spine (Phila Pa 1976). 2000;25(18):2358-63. 12.van Rhijn LW, Plasmans CM, Veraart BE. No relationship exists between the correction of the thoracic and the lumbar curves after selective thoracic fusion for adolescent idiopathic scoliosis King type II. Eur Spine J. 2002;11(6):550-5.

13.Jansen RC, van Rhijn LW, Duinkerke E, van Ooij A. Predictability of the spontaneous lumbar curve correction after selective thoracic fusion in idiopathic scoliosis. Eur Spine J. 2007;16(9):1335-42.

14.Lenke LG, Betz RR, Harms J, Bridwell KH, Clements DH, Lowe TG, et al. Adolescent idiopathic scoliosis: a new classification to determine extent of spinal arthrodesis. J Bone Joint Surg Am. 2001;83A(8):1169-81. Comment in: J Bone Joint Surg Am. 2002;84-A(6):1080-1; author reply 1081. J Bone Joint Surg Am. 2002;84-A(5):870; author reply 870-1.

15.Cobb JR. Outline for the study of scoliosis. Instr Course Lect. 1948;5:261-75.

16. Richards BS, Scaduto A, Vanderhave K, Browne R. Assessment of trunk balance in thoracic scoliosis. Spine (Phila Pa 1976). 2005;30(14):1621-6.

17. Winter RB, Lonstein JE. A metaanalysis of the literature on the issue of selective thoracic fusion for the King-Moe type II curve pattern in adolescent idiopathic scoliosis. Spine (Phila Pa 1976). 2003;28(9):948-52. 
18.Lenke LG, Betz RR, Bridwell KH, Harms J, Clements DH, Lowe TG. Spontaneous lumbar curve coronal correction after selective anterior or posterior thoracic fusion in adolescent idiopathic scoliosis. Spine (Phila Pa 1976). 1999;24(16):1663-71; discussion 1672.
19.Suk S, Lee SM, Chung ER, Kim JH, Kim SS. Selective thoracic fusion with segmental pedicle screw fixation in the treatment of thoracic idiopathic scoliosis: more than 5-year follow-up. Spine (Phila Pa 1976). 2005;30(14):1602-9.

20. Ogilvie JW. The "S" curve, or, when is selective thoracic fusion indicated? Coluna/Columna. 2006;5(4):280-1.

\section{Correspondência}

Robert Meves

Departamento de Ortopedia e Traumatologia, Pavilhão Fernando Simonsen

Rua Cesário Motta Júnior, 112

CEP: $01221-020$ - São Paulo (SP), Brasil

Tel.: (1 1) 2176-7000, ramal 1565

E-mail: robertmeves@hotmail.com 